\title{
Wettability alteration and Oil- Water-Rock Interaction at Hydrated Kaolinite Clay Mineral Surfaces
}

\author{
Rixin Zhao ${ }^{1}$, Shuangfang $\mathrm{Lu}^{1 *}$, Haitao Xue ${ }^{1}$ \\ ${ }^{1}$ School of Geosciences, China University of Petroleum (East \\ China), Qingdao 266580, China (*correspondence: \\ lushuangfang@upc.edu.cn)
}

In this study, equilibrium molecular dynamics simulations have been used to understand the interactions and wettability alteration mechanism of oil components and water at the hydrated surfaces of the clay mineral kaolinite. N-Hexane, $\mathrm{H}_{2} \mathrm{O}$, N-Octadecylic Acid and Asphaltene have been modelled at both the hydroxylated and silicate surfaces of kaolinite. Additionally, the mechanism of mineral surface wettability alteration by the polar oil components is observed via the interaction energy[1].
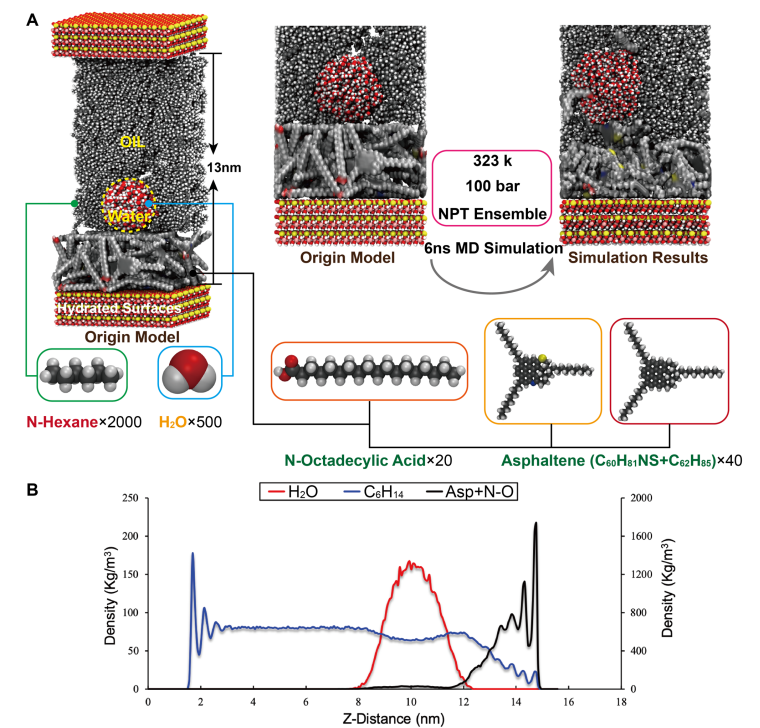

Figure 1: MD simulation schematics and density distribution diagrams

The key results show that the polar oil components can alter the wettability from water-wet toward more oil-wet conditions on the mineral surfaces. We also manifest that the polarity of oil molecules can affect the water contact angles on hydrated surfaces. Furthermore, the van der Waals interactions were found to provide the largest contribution for driving the adsorption of nonhydrocarbon and asphaltene on mineral surfaces leads to wettability alteration.

[1]Xiong Y et al. (2017) J PHYS CHEM C, 121, 5020-5028. 